\title{
Stereotyped Odor-Evoked Activity in the Mushroom Body of Drosophila Revealed by Green Fluorescent Protein-Based $\mathrm{Ca}^{2+}$ Imaging
}

\author{
Yalin Wang, ${ }^{1}$ Hui-Fu Guo, ${ }^{1}$ Thomas A. Pologruto, ${ }^{1,2,3}$ Frances Hannan, ${ }^{1}$ Inessa Hakker, ${ }^{1}$ Karel Svoboda,,${ }^{1,2}$ and Yi Zhong ${ }^{1}$ \\ ${ }^{1}$ Cold Spring Harbor Laboratory, Cold Spring Harbor, New York 11724, and ${ }^{2}$ Howard Hughes Medical Institute and ${ }^{3}$ Graduate Program in Biophysics, \\ Harvard University, Boston, Massachusetts 02138
}

\begin{abstract}
To study the representation of olfactory information in higher brain centers, we expressed a green fluorescent protein-based $\mathrm{Ca}^{2+}$ sensor, G-CaMP, in the Drosophila mushroom body (MB). Using two-photon microscopy, we imaged odor-evoked G-CaMP fluorescence transients in MB neurons [Kenyon cells (KCs)] with single-cell resolution. Odors produced large fluorescence transients in a subset of KC somata and in restricted regions of the calyx, the neuropil of the MB. In different KCs, odor-evoked fluorescence transients showed diverse changes with odor concentration: in some KCs, fluorescence transients were evoked by an odor at concentrations spanning several orders of magnitude, whereas in others only at a narrow concentration range. Different odors produced fluorescence transients in different subsets of KCs. The spatial distributions of KCs showing fluorescence transients evoked by a given odor were similar across individuals. For some odors, individual KCs with fluorescence transients evoked by a particular odor could be found in similar locations in different flies with spatial precisions on the order of the size of $\mathrm{KC}$ somata. These results indicate that odor-evoked activity can have remarkable spatial specificity in the $\mathrm{MB}$.
\end{abstract}

Key words: odor; stereotype; mushroom body; calcium imaging; G-CaMP; two photon; Kenyon cell

\section{Introduction}

Growing evidence indicates a stereotypical anatomical and functional organization in early stages of the olfactory pathway of insects and vertebrates (Hildebrand and Shepherd, 1997; Buck, 2000; BozzaandMombaerts, 2001; KauerandWhite, 2001; Korsching, 2002). In both insects and vertebrates, each olfactory receptor neuron (ORNs) likely expresses only one specific olfactory receptor (OR) gene (Chess et al., 1994; Ressler et al., 1994; Clyne et al., 1999; Malnic et al., 1999; Vosshall et al., 1999, 2000), and ORNs expressing the same ORs project to spatially invariant glomeruli in the antennal lobe in insects (Gao et al., 2000; Vosshall et al., 2000) and olfactory bulb in vertebrates (Mombaerts et al., 1996). Each odor may activate a combination of ORs and therefore ORNs (Malnic et al., 1999; de Bruyne et al., 2001). The combinatorial activation of ORs and the precise anatomical convergence of ORN projections give rise to topographic activation patterns of glomeruli that are both odor specific and conserved in different individuals (Stocker et al., 1983; Rodrigues, 1988; Johnson et al., 1998; Galizia et al., 1999; Rubin and Katz, 1999; Wachowiak and Cohen, 2001; Wang et al., 2003).

Received Aug. 10, 2003; revised June 1, 2004; accepted June 3, 2004.

This work was supported by grants from the National Institutes of Health (K.S., Y.Z.) and United States Army (Y.Z.) and by the Howard Hughes Medical Institute (K.S.). We thank Junichi Nakai for providing the G-CaMP plasmid; Jing W. Wang for discussion of results; Tim Tully, Ann-shyn Chiang, and Robert Brandt for help using Amira; and Gengxin Chen for help with data analysis.

Correspondence should be addressed to Yi Zhong, Department of Neuroscience, Cold Spring Harbor Laboratory, 301 Beckman, 1 Bungtown Road, Cold Spring Harbor, NY 11724. E-mail: zhongyi@cshl.org.

DOI:10.1523/JNEUROSCI.3727-03.2004

Copyright $\odot 2004$ Society for Neuroscience $\quad$ 0270-6474/04/246507-08\$15.00/0
How are neurons in higher olfactory centers organized to respond to odor stimulation? Is the topographic nature of the odorspecific glomerular activation retained in higher-order olfactory centers? Addressing such questions is critical for our understanding of the functional organization of high-order brain centers and olfactory coding. In Drosophila, single-cell tracing has revealed a stereotyped "axon map" of projection neurons. Projection neurons innervating the same glomeurlus in the antennal lobe exhibit a strikingly similar axonal branching pattern in the protocerebrum, whereas those corresponding to different glomeruli differ (Marin et al., 2002; Wong et al., 2002). Furthermore, transneuronal tracer experiments in mice show that ORNs that express the same OR are targeted to specific clusters of thousands of olfactory cortical neurons, and that the locations of these clusters are conserved in different individuals (Zou et al., 2001). These findings suggest that a stereotyped organization may exist in the higher-order olfactory centers reflecting those in the antennal lobe and olfactory bulb.

We previously reported odor-specific spatial patterns of activity in the Drosophila mushroom body (MB) (Wang et al., 2001). However, that study used bulk labeling with $\mathrm{Ca}^{2+}$-sensitive dyes and wide-field fluorescence imaging, which do not allow adequate spatial resolution to detect the activity in individual neurons. Furthermore, the variability of dye loading in the living fly brain complicates comparisons of the spatial activity patterns between individual flies. To overcome these limitations, we developed a transgenic fly with targeted expression of a green fluorescence protein (GFP)-based $\mathrm{Ca}^{2+}$ sensor (G-CaMP) (Nakai et 
al., 2001) in the MB. Using two-photon imaging (Denk and Svoboda, 1997), we recorded odor-evoked G-CaMP fluorescence transients in the MB with single-cell resolution. Exposure of the fly to odors resulted in a large increase in G-CaMP fluorescence intensity in specific groups of Kenyon cells (KCs), reflecting intracellular $\mathrm{Ca}^{2+}$ elevations in these neurons. KCs showing fluorescence transients evoked by a specific odor resided at similar locations of the brain across individual flies.

\section{Materials and Methods}

G-CaMP construct and transformation. The insert from the pN1-G-CaMP1.3 plasmid (Nakai et al., 2001) was cut out with BglII and NotI and inserted into the pUAST vector (Brand and Perrimon, 1993). The resulting construct UAS-GCaMP was injected into $2202 \mathrm{u}$ embryos together with pTurbo (encoding transposase) to generate germ line transformants (Spradling and Rubin, 1982). The resulting transformants were crossed to an MB-specific GAL4 driver line, OK107 (Connolly et al., 1996), to examine the expression of G-CaMP. OK107 has the strongest expression in the MB among all GAL4 drivers. Forty-seven transformation lines were shown to have fluorescence, and the one with the strongest expression was chosen for the study reported here.

Optical recording in living flies. Living flies were prepared for MB imaging as described previously (Wang et al., 2001). Briefly, a fly was held in a truncated pipette tip, exposing only the head. A tiny window was cut in the head to reveal the $\mathrm{KC}$ soma layer, and a drop of adult fly saline [115 $\mathrm{mm} \mathrm{NaCl}, 5 \mathrm{~mm} \mathrm{KCl}, 6 \mathrm{~mm}$ $\mathrm{CaCl}_{2} \cdot 2 \mathrm{H}_{2} \mathrm{O}, 1 \mathrm{~mm} \mathrm{MgCl} \cdot 6 \mathrm{H}_{2} \mathrm{O}, 4 \mathrm{~mm}$ $\mathrm{NaHCO}_{3}, 1 \mathrm{mM} \mathrm{NaH} \mathrm{PO}_{4} \cdot 1 \mathrm{H}_{2} \mathrm{O}, 5 \mathrm{~mm}$ trehalose, $75 \mathrm{~mm}$ sucrose, and $5 \mathrm{~mm} \mathrm{~N}$-Tris (hydroxymethyl) methyl-2-aminoethanesulfonic acid, $\mathrm{pH}$ $7.1,356 \mathrm{mOsm}]$ was placed immediately over the opening. The exposed brain region was made accessible to the water immersion lens [63X, 0.9 numerical aperture (NA); Olympus America, Melville, NY] by placing a coverslip with a small window matched to the head opening over the head.

Imaging with wide-field fluorescence microscope was as described previously (Wang et al., 2001). Fluorescence images $(128 \times 128$ pixels; pixel size, $1.1 \times 1.1 \mu \mathrm{m})$ were acquired using a CCD camera (20 frames per second).

For two-photon imaging, a custom-built two-photon laser scanning microscope was used as described previously (Lendvai et al., 2000). The objective was from Zeiss ( $40 \times, 0.8 \mathrm{NA}$; Zeiss, Thornwood, NY). Fluorescence was detected in whole-field detection mode. Microscope control was accomplished using the custom-written software package ScanImage (Pologruto et al., 2003). The light source was a Ti-Sapphire laser (Spectra-Physics, Mountain View, CA) running at a wavelength of $\sim 910$ $\mathrm{nm}$. Images $(512 \times 512$ pixels; pixel size, $0.27 \times 0.27 \mu \mathrm{m})$ were acquired at a rate of approximately two frames per second.

Odor delivery. Odors were delivered to the fly through a micropipette located $\sim 2 \mathrm{~mm}$ from the antennae by switching from a constant air stream bubbled through mineral oil to an odor stream, which was produced by bubbling air through mineral oil containing an odor. Odor was applied for $2 \mathrm{sec}$ before switching back to the air stream. An air control was performed following the same procedure but with the odor stream medial is to the right, throughout.
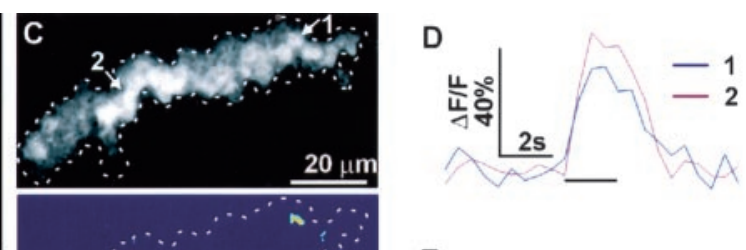

$100 \mu \mathrm{m}$

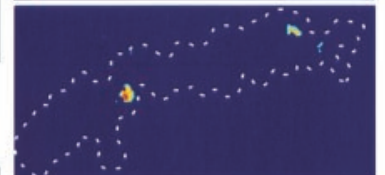

E
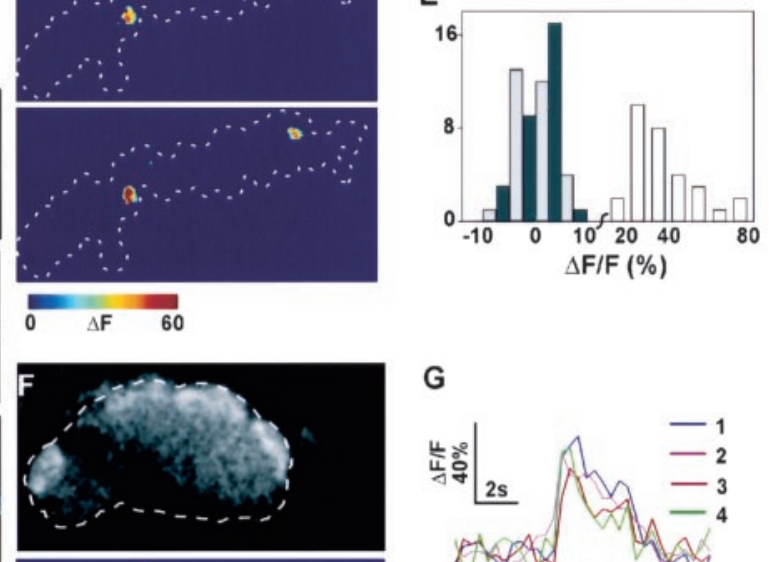

G
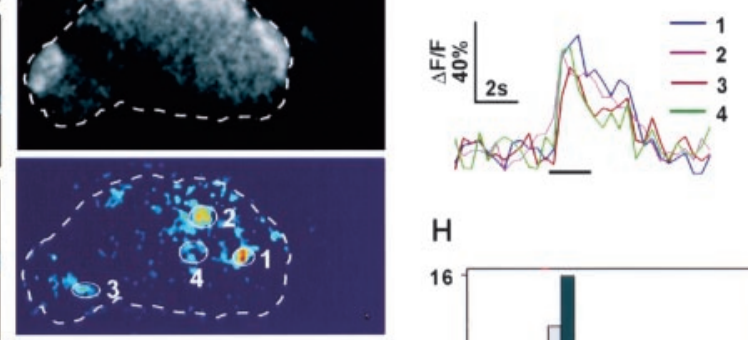

$\mathrm{H}$

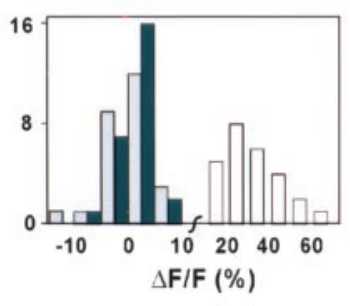

Figure 1. Imaging of odor-evoked G-CaMP fluorescence transients in Drosophila MBs. A, G-CaMP fluorescence in the MB of a living fly. G-CaMP expression clearly identifies the MB structure. $B$, Two-photon images of the boxed region in $A$ at different depths showing the KC somata (top three panels) and calyx regions (bottom two panels) (depth, 0 corresponds to the top of the soma ( recorded regions. $D, G$, Time courses of fluorescence transients in response to EA stimulation in single neurons $(D)$ and calycal areas $(G)$ (regions of interest indicated in $($ and $F$ ); horizontal bars indicate the duration of the odor stimuli. $E$, H, Histograms of the peak amplitudes of the fluorescence transients in the odor-responsive $K C$ somata $(E)$ and calycal areas $(H)$, in response to $E A$ (white dent's $t$ test). There was no significant difference between activities from randomly chosen nonresponsive neurons or areas and air control level. Data were collected from $30 \mathrm{KC}$ somata and calycal areas from 23 individual flies. Anterior is to the bottom, and

replaced by a second air stream. Air controls were interleaved with odor stimulus trials. A specific concentration of an odor was achieved by diluting the pure chemical odorant in mineral oil. A concentration of $10^{-3}$ corresponded to a 1000-fold dilution. We measured the final odor concentrations with a flame ionization detector (MicroFID; Pine Environmental, Cranbury, NJ) and found there was no attenuation of odor concentration from the outlet of the micropipette to the position of antennae. For ethyl acetate, a concentration of $10^{-3}$ was $\sim 1.2 \%$ of the saturated vapor, and other concentrations were proportional to the dilutions made. There was a 4 min interval between successive odor stimulations to prevent habituation (Skoulakis and Acevedo, 2003). At such an interval, responses in the MB to odor stimuli were not affected by the preceding odor exposure.

Data analysis. Imaging data were analyzed using a custom program written in MatLab (MathWorks, Natick, MA). $\Delta F$ images were obtained by subtracting the mean of the background fluorescence, which was determined by averaging 10 frames before the stimulus, from the average of 3-4 frames at the peak of the response. We searched for regions of the 
A
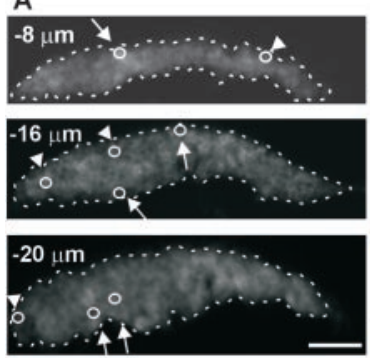

B

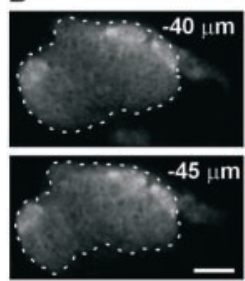

C
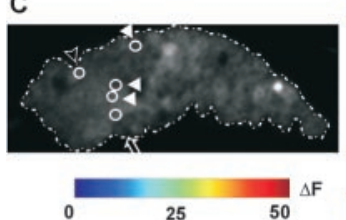

25
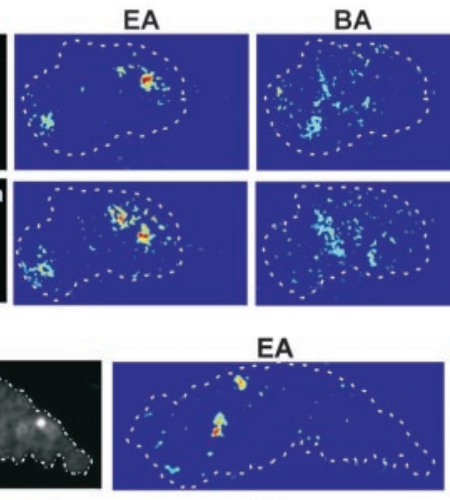

EP

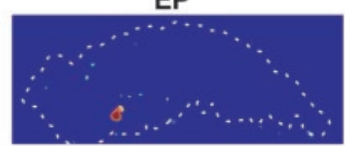

BA
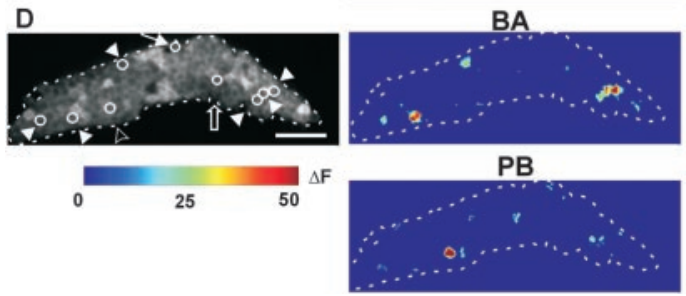

EA

BA
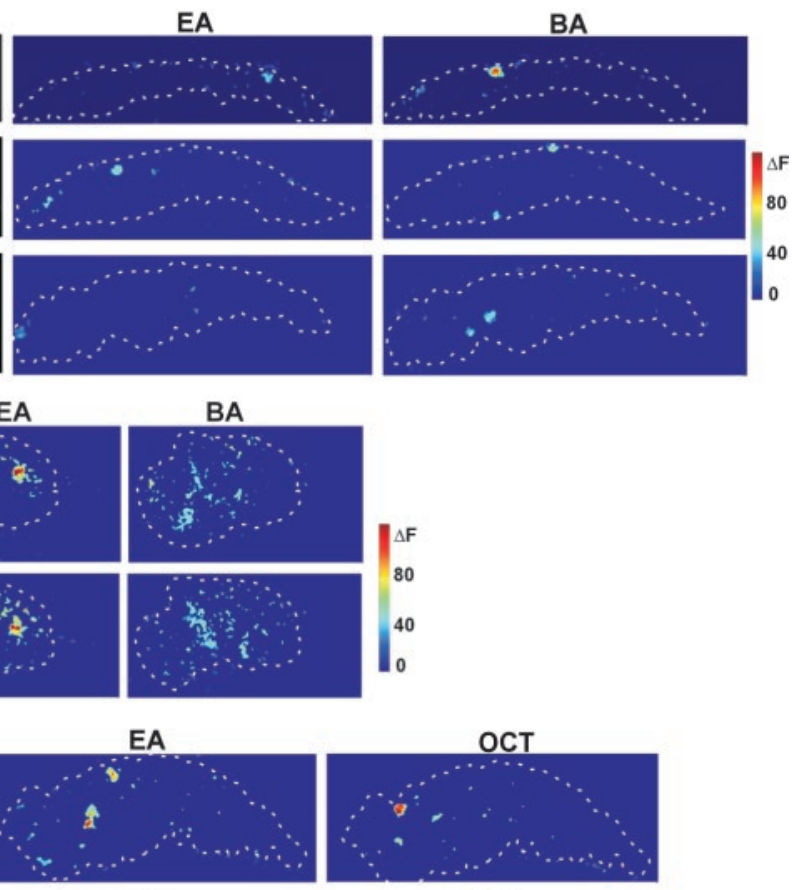

CIN
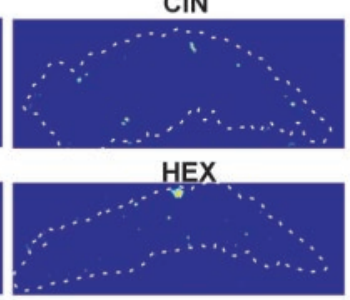

EO

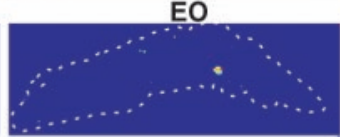

Figure 2. G-CaMP responses to different odors in the MB. $A, B, \mathrm{G}$-CaMP responses to stimuli by $\mathrm{EA}$ and benzaldehyde (BA) recorded in $K C$ soma layer $(A)$ and calyx $(B)$ of a single fly at indicated depths. $K C$ s with fluorescence transients evoked by $E A$ (arrowheads) and BA (arrows) are indicated by circles in gray scale panels on the left that show the structure of the imaged optical sections. Fluorescence increases in the activated neurons and calycal areas ranged from 22.1 to $102.5 \%$. C, D, G-CaMP responses to stimuli by multiple odors in the soma layer (depth, $-20 \mu \mathrm{m}$ ) recorded from two individual flies. KCs with fluorescence transients elicited by $B A$ (white arrowheads), cineole (CIN), EA (white arrowheads), ethyl octanoate (E0; black-filled arrow), ethyl propionate (EP; black-filled arrow), hexanol (HEX; white arrow), OCT (black-filled arrowhead), and propyl butyrate (PB; black-filled arrowhead) are indicated by circles in the left gray scale panels. The range of fluorescence increases in the identified neurons was from 21.5 to $81.3 \%$. Scale bars, $20 \mu \mathrm{m}$. Orientation is as in Figure 1.

MB showing odor-evoked G-CaMP fluorescence transients that were significantly above the control activity level. Two criteria were used. First, for individual pixels, the fluorescence intensity change had to exceed the background fluorescence fluctuations. Background fluorescence fluctuations were calculated by subtracting the average of 10 frames preceding the stimulus from each of the 10 frames. Then, the mean and SEM of the fluctuation were obtained for each pixel. A threshold was then applied to each pixel of the $\Delta F$ image. We found that a threshold corresponding to $3 \times$ SEM was optimal: this threshold excluded $>80 \%$ of the fluorescence signals that were not distinguishable from air controls without exclusion of signals $(<5 \%)$ as judged by other measures (supplemental Fig. $1 B$, available at www.jneurosci.org/cgi/content/full/24/29/6507/DC1). However, not everything appearing in the $\Delta F$ image after such thresholding could be considered as odor-evoked activity. Occasionally, switching of the air flow could produce apparent fluorescence changes (supplemental Fig. 1C, available at www.jneurosci.org/cgi/content/full/24/29/ $6507 / D C 1)$. Therefore, a second criterion was established: fluorescence changes $(\Delta F / F)$ in regions corresponding to $\mathrm{KC}$ somata were scored only if they exceeded (Student's $t$ test; $p<0.001$ ) the air control level (air

controls were performed after each odor stimulus trial by measuring fluorescence changes in response to an air stream).

Three-dimensional reconstruction. After recording odor responses, the $\mathrm{MB}$ was imaged from top to bottom ( $\mathrm{z}$-step $=-1 \mu \mathrm{m})$, where the peduncle begins to emerge, and a threedimensional $\mathrm{MB}$ structure was constructed with Amira (TGS, San Diego, CA). Neurons with odor-evoked G-CaMP fluorescence transients were mapped onto the MB structure. Wire frames were used to illustrate the reconstructed MBs to make the KCs inside visible to the viewer. The orientation of MBs from different flies were aligned based on the positions of the protrusion emanating from the calyx as well as the orientation of the peduncle and four axon bundles that emerge within the calyx (see Fig. 4A).

For quantitative comparison of the distributions of KCs displaying odor-evoked G-CaMP fluorescence transients across individual flies, each MB was scaled to a template with Amira. The template was an $\mathrm{MB}$ with dimensions closest to the average of all MBs being compared. The coordinates of KCs in the scaled MB were obtained with Amira and plotted in SigmaPlot (Systat Software, Richmond, CA). The mean distance between a cluster of KCs and the corresponding centroid was determined using Excel (Microsoft, Seattle, WA).

\section{Results}

We used the GAL4 enhancer trap system to target the expression of G-CaMP in MBs (Fig. 1 $A, B$ ). OK107, a GAL4 line that has expression in most of the MB neurons, was used to drive G-CaMP expression (Connolly et al., 1996). In flies that had both OK107 and G-CaMP transgenes, the basal level of G-CaMP fluorescence allowed clear identification of the $\mathrm{MB}$, which was accessible for imaging from the top of the head. The part of the MB examined consisted of the KC soma layer (30-35 $\mu \mathrm{m}$ thick), containing $\sim 2500$ densely packed KCs per brain hemisphere (Technau and Heisenberg, 1982), and the calyx ( $\sim 30 \mu \mathrm{m}$ thick), which lay below the soma layer. Delivery of an air-borne odor, ethyl acetate (EA), to a living fly elicited G-CaMP fluorescence transients in both $\mathrm{KC}$ soma layer and the calyx (Fig. $1 C, F$ ), reflecting activitydependent increases in cytoplasmic $\mathrm{Ca}^{2+}$ concentration (Svoboda et al., 1997; Nakai et al., 2001; Wang et al., 2003; Yu et al., 2003). The increase in G-CaMP fluorescence could reach $100 \%$. Odor-evoked fluorescence transients overlapped spatially with individual KC somata (Fig. 1C). Odor-evoked fluorescence transients were highly reproducible (Fig. 1C,F). The time courses of odor-evoked fluorescence transients were similar in different $\mathrm{KC}$ somata and different areas of the calyx (Fig. 1D, G) and across trials (Fig. 1C,F). Fluorescence transients reported in this study referred only to those significantly above the control level activity (see Materials and Methods) (Fig. $1 E, H$; supplemental Fig. $1 D$, available at www.jneurosci.org/cgi/content/full/24/29/ 6507/DC1).

Different odorants evoked fluorescence transients in distinct 
subpopulations of KCs. Recordings at different depths in the soma layer showed that EA and benzaldehyde evoked fluorescence transients from different KCs at every optical section examined ( $n=16$ flies). Examples of recordings from three sections in a fly are shown in Figure $2 A$. The finding in the $\mathrm{KC}$ soma layer was consistent with recordings in the calyx, where EA and benzaldehyde induced distinct spatial patterns of fluorescence activity (Fig. $2 B$ ). Most benzaldehyde-evoked activity was in the center of the calyx, whereas EA-evoked activity was mostly in the medial and lateral regions.

The specificity of KC fluorescence transients in response to particular odors was further analyzed by recording responses to eight odors in the soma layer at a depth of $-20 \mu \mathrm{m}$ (Fig. 2C,D). Each odor evoked fluorescence transients in distinct groups of KCs. Although EA and benzaldehyde elicited fluorescence transients in several $\mathrm{KCs}$, most other odors caused G-CaMP responses only in one $\mathrm{KC}$, whereas no significant signal was detected for cineole at this location.

We examined fluorescence transients in the $\mathrm{MB}$ in response to odors at different concentrations. KCs with odor-evoked fluorescence transients showed diverse degrees of dependency on odor concentration (Fig. 3). In some KCs (23 of 64 EAresponsive $\mathrm{KCs}$ recorded), G-CaMP fluorescence transients were elicited by a broad range of odor concentrations that spanned several orders of magnitude (Fig. $3 A$, white arrowheads point to $\mathrm{KCs}$ ). The amplitudes of EA-evoked fluorescence transients in these $\mathrm{KCs}$ varied to different concentrations of EA (Fig. $3 B$ ). In other KCs (41 of 64 EA-responsive KCs recorded), fluorescence transients were only elicited by a narrow concentration range of odors (Fig. $3 A$, arrows and black-filled arrowheads point to $\mathrm{KCs}$ ). The diverse concentration dependency was not the result of trial-to-trial variability. Repetitive stimulation with the same concentration series evoked consistent fluorescence transients in the same KCs (Fig. 3A, compare left and right panels).

The diverse concentration dependency was also observed in the KC dendritic region, the calyx. Calycal fluorescence transients were evoked either by broad ( 10 of 29 responsive areas) or narrow ( 19 of 29 responsive areas) odor concentration ranges. For example, in some calycal areas (Fig. $3 C, D$, area 1), fluorescence transients were evoked by EA at all four concentrations tested, spanning from $10^{-5}$ to $10^{-2}$. Meanwhile, there were areas in which fluorescence transients were detected only at a specific concentration (Fig. 3C,D, area 3 ) or at two different concentrations (Fig. $3 C, D$, areas 2 and 4$)$.

To quantify the spatial distribution of KCs with odor-evoked fluorescence transients, we reconstructed the positions of the $\mathrm{KCs}$ in the MB. Typically, eight responses were recorded per fly and odor in the soma layer from optical sections located 2-4 $\mu \mathrm{m}$ apart. KCs with odor-evoked fluorescence transients were
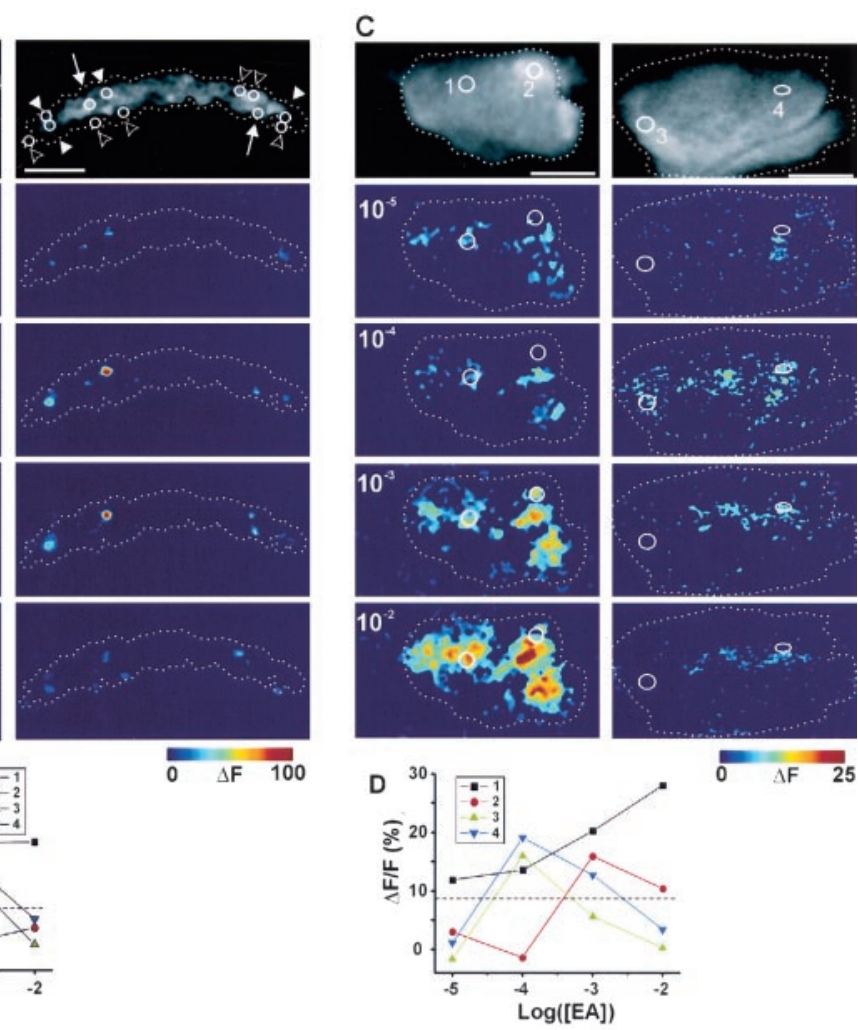

Figure 3. Dependence on odor concentration. A, Fluorescence activities in the KC soma layer in response to four different concentrations of EA. Positions of the KCs with EA-evoked fluorescence transients are indicated with circles in the top panel of each column that shows the structure of the imaged optical section. The left column shows a series of recordings performed in sequence 政 concentrations, whereas those pointed by black-filled arrowheads and white arrows displayed fluorescence transients in response respectively. Fluorescence increases in activated regions ranged from 11.4 to $72.7 \%$. D, Peak fluorescence activities from the calycal areas indicated in C. The dashed line in B and D shows the top limit of the control level activities (mean \pm 3 SDs). Scale bars, $20 \mu \mathrm{m}$. Orientation of the images is as in Figure 1.

mapped to the corresponding reconstructed $\mathrm{MB}$ structure (Fig. $4 A)$. Visual inspection revealed that EA and 3-octanol (OCT) evoked fluorescence transients in distinct sets of KCs that were located in different quadrants of the $\mathrm{MB}$ (Fig. $4 B, C$ ). KCs with OCT-evoked fluorescence transients were clustered in the central area, whereas KCs with EA-evoked fluorescence transients were localized primarily in two large clusters located laterally and medially.

To compare the distribution patterns of KCs with odor-evoked fluorescence transients across flies, we normalized the MBs to a template using a similar approach for constructing the standard Drosophila brain (Rein et al., 2002) and the coordinates of the KC somata along the dorsoventral, mediolateral, and anteroposterior axes were obtained. It was more evident in the normalized MB that EA and OCT evoked fluorescence transients in distinct sets of KCs, which spatial distributions were similar in different individuals. For example, KCs with fluorescence transients evoked by EA and OCT occupied different regions (Fig. 4D), and KCs with fluorescence transients evoked by the same odor from different flies were distributed in similar locations in the normalized MB (Fig. 4E,F).

The conserved odor-specific distribution patterns were further demonstrated by quantitative comparison of the distribu- 
A
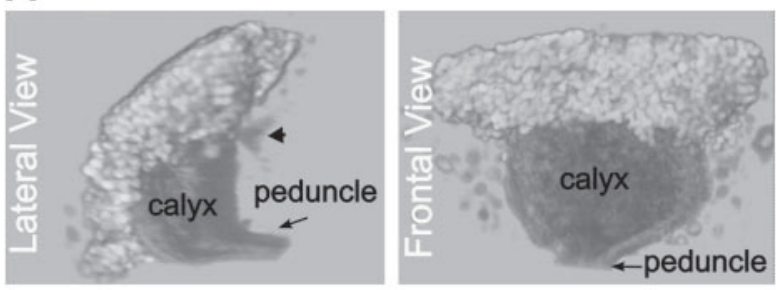

B
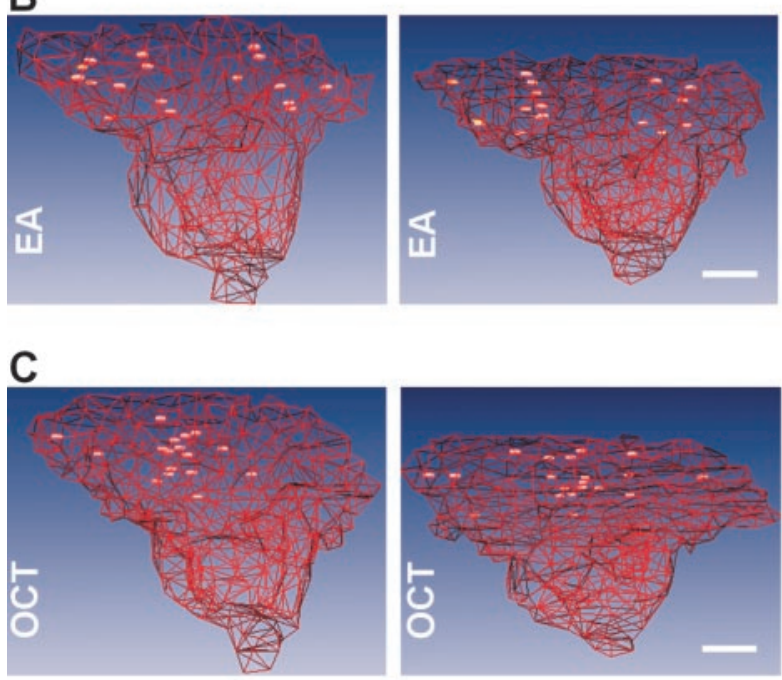

$\mathbf{F}$

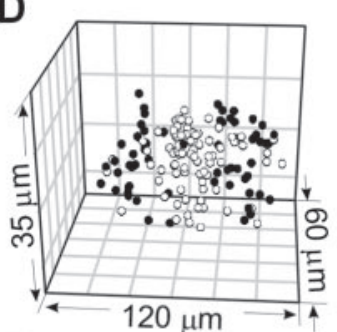

E
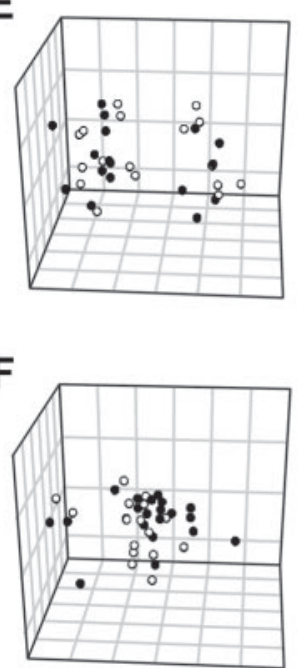

Figure 4. Spatial distribution of KCs with fluorescence transients evoked by EA and OCT. A, Two reconstructed views of the part of the MB examined by two-photon imaging. In the lateral view, the arrowhead points to a protrusion from the calyx in which identity is unknown. To make the KCs inside the MB visible, reconstructed MBs are shown with wire frames in following figures. $B$, $C$, Spatial distribution of KCs with fluorescence transients evoked by EA ( $B)$ and OCT ( $C$ ). Frontal views of two representative reconstructed $M B$ structures are shown for each odor. Medial is to the right. $D, K C$ s with fluorescence transients evoked by $E A(O)$ and OCT (O) occupy different regions when plotted in a normalized MB. Data were collected from five individual flies for each odor. $E$, KCs with EA-evoked fluorescence transients from the two flies shown in $B$ are located in similar regions in the normalized MB. F, KCs with OCT-evoked fluorescence transients from the two flies shown in Clocate in similar places in the normalized MB. Dimensions of the normalized MBs in $E$ and $F$ are as in D. Scale bars, $20 \mu \mathrm{m}$.

tions of KCs with EA- and OCT-evoked fluorescence transients along the mediolateral axis in different flies (Table 1). The normalized $\mathrm{MB}$ was evenly divided into six segments along the mediolateral axis, and distributions of KCs with EA- and OCTevoked fluorescence transients in these segments from different flies were compared. The correlation coefficients were low for inter-odor comparisons $(-0.20 \pm 0.20$, between KCs with EAand OCT-evoked fluorescence transients) and were high for intra-odor comparisons $(0.67 \pm 0.19$ and $0.84 \pm 0.12$, for KCs with fluorescence transients evoked by EA and OCT from five flies, respectively). This demonstrated that the overall distribution patterns of the $\mathrm{KC}$ somata with fluorescence transients evoked by EA and OCT were both odor specific and conserved across individual flies and therefore stereotyped.

We next asked whether this stereotypy might exist at the single-neuron level. However, the number of KCs involved in G-CaMP fluorescence responses to EA and OCT made it difficult to compare the distribution of single KCs from fly to fly. An ideal case would be if there were only a few neurons involved in response to an odor. Electrophysiological recordings have shown that several odors including cineole and 4-methyl cyclohexanol $(\mathrm{MCH})$ fail to elicit any activity from 16 functional ORNs in the basiconic sensila on the antennae (de Bruyne et al., 2001), raising the possibility that they may acti-

vate few KCs. We therefore examined GCaMP fluorescence in the $\mathrm{MB}$ in response to cineole and $\mathrm{MCH}$.

Recordings from optical sections separated by $2-4 \mu \mathrm{m}$ revealed that very few KCs displayed fluorescence transients in response to both cineole $(n=17$ recordings from 3 flies $)$ and $\mathrm{MCH}(n=16$ recordings from 3 flies). The KCs with odorevoked fluorescence transients appeared primarily in the top and middle portions of the soma layer for cineole and $\mathrm{MCH}$, respectively. High-resolution measurements (sections 1-2 $\mu \mathrm{m}$ apart) in these subregions of the soma layer were performed to uncover KCs displaying fluorescence transients evoked by cineole and $\mathrm{MCH}$. For cineole, four of six flies examined had three such KCs each, and the other two had four each (Fig. 5A-D). When reconstructed in the normalized MB for all six flies, three of the KCs with cineole-evoked fluorescence transients (Fig. $5 A-D, \mathrm{a}, \mathrm{b}, \mathrm{c}$ ) formed three corresponding clusters (Fig. $5 E$ ). In each cluster, the mean distance of the KCs from the corresponding centroid was $3.4 \pm 1.5,4.3 \pm$ 2.1 , and $3.8 \pm 1.8 \mu \mathrm{m}$ for clusters $\mathrm{a}, \mathrm{b}$, and $c$, respectively. The fourth neuron, shown in two of the six flies (Fig. 5C,D), appeared in variable positions with respect to the other three neurons. For $\mathrm{MCH}$, three flies examined each had two KCs displaying $\mathrm{MCH}$-evoked fluorescence transients, and two others had one each (Fig. $5 F-I$ ). These neurons also formed tight clusters when plotted in the normalized MB (Fig. 5J), the mean distances of neurons in each cluster to the corresponding centroid were $3.8 \pm$ 2.0 and $3.9 \pm 2.8 \mu \mathrm{m}$. These results revealed a striking degree of stereotypy in the distribution of KCs with odor-evoked fluorescence transients at single-neuronal level: a KC displaying fluorescence transients evoked by cineole or $\mathrm{MCH}$ may be found in different individuals at a similar position in the $\mathrm{MB}$ with a spatial precision on the order of the size of one or two KC somata ( $3.9 \mu \mathrm{m})$.

The stereotyped distribution of KCs displaying odor-evoked fluorescence transients is consistent with stereotyped distribution of G-CaMP fluorescence activities in the calyx, revealed by recordings with wide-field fluorescence microscopy. The limited effective spatial resolution of this technique in thick tissues is a minor disadvantage in imaging the calyx, because the distribution of odor-evoked G-CaMP fluorescence activities is relatively diffuse (Figs. 1F, 2). Different odors evoked distribution patterns of fluorescence transients in the calyx that were odor specific and conserved across flies (Fig. 6, Table 2). The basic features of EAand benzaldehyde-stimulated distribution patterns of G-CaMP fluorescence activity revealed by two-photon imaging were preserved when imaged with wide-field microscopy. EA-induced fluorescence activity was localized medially and laterally, whereas benzaldehyde-induced was distributed in the middle part of the calyx. 
Table 1. Comparison of distributions of KCs with fluorescence transients evoked by EA and OCT from different flies along the mediolateral dimension

\begin{tabular}{|c|c|c|c|c|c|c|c|c|c|c|}
\hline & EA1 & $\mathrm{EA} 2$ & $\mathrm{EA3}$ & EA4 & EA5 & 0CT1 & OCT2 & OCT3 & OCT4 & 0CT5 \\
\hline EA1 & & 0.68 & 0.93 & 0.46 & 0.94 & -0.03 & -0.29 & -0.49 & 0.16 & -0.34 \\
\hline EA2 & & & 0.43 & 0.66 & 0.71 & -0.02 & -0.22 & -0.21 & -0.13 & 0.02 \\
\hline EA3 & & & & 0.48 & 0.83 & -0.11 & -0.29 & -0.51 & 0.15 & -0.44 \\
\hline EA4 & & & & & 0.52 & -0.37 & -0.29 & -0.26 & -0.36 & -0.17 \\
\hline EA5 & & & & & & -0.04 & -0.20 & -0.37 & 0.19 & -0.26 \\
\hline 0CT1 & & & & & & & 0.90 & 0.81 & 0.89 & 0.86 \\
\hline OCT2 & & & & & & & & 0.96 & 0.84 & 0.92 \\
\hline OCT3 & & & & & & & & & 0.65 & 0.96 \\
\hline OCT4 & & & & & & & & & & 0.63 \\
\hline OCT5 & & & & & & & & & & \\
\hline
\end{tabular}

The normalized MBs were each divided evenly into six segments along the mediolateral axis. Distributions of KCs with fluorescence transients evoked by EA and OCT in these segments from 10 different flies (five for EA and five for OCT) were compared, and Pearson's product moment correlation coefficients were generated.

\section{Discussion}

The targeted expression of the GFP-based $\mathrm{Ca}^{2+}$ sensor G-CaMP, in combination with two-photon imaging, has allowed us to examine odor-evoked responses in the $\mathrm{MB}$ at the single-neuron level. We observed large fluorescence transients in the calyx and in individual KC somata in response to odor stimulation. Different odors evoked G-CaMP responses in different sets of KCs with diverse dependencies on odor concentrations. The spatial distribution of KCs displaying odor-evoked fluorescence transients (in somata and dendritic region) was stereotyped across flies.

Genetically encoded $\mathrm{Ca}^{2+}$ reporters have been used to map odor-evoked activities in the Drosophila antennal lobe (Wang et al., 2003) and to measure $\mathrm{Ca}^{2+}$ responses in $\mathrm{KCs}$ induced by extracellular application of high potassium or acetylcholine (Yu et al., 2003). In our studies, G-CaMP fluorescence transients in the calyx and somata of KCs likely reflect $\mathrm{Ca}^{2+}$ entry through synaptic nicotinic acetyl-
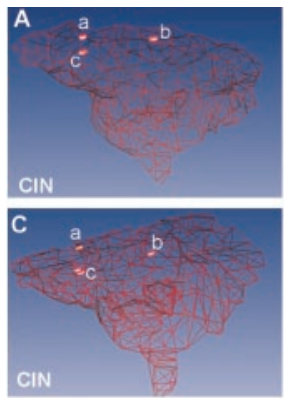

E

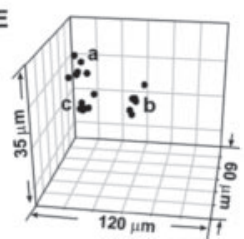

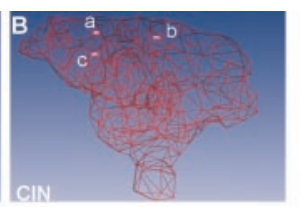
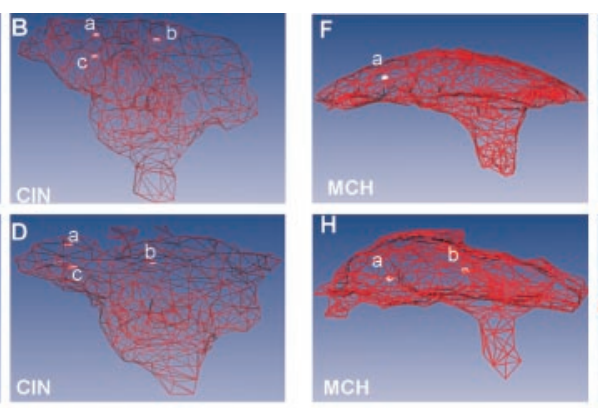

J

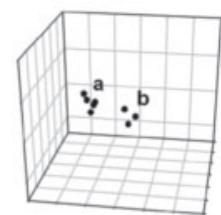

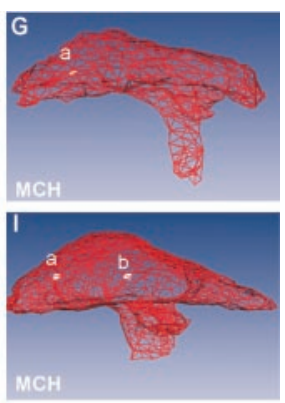

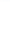

Figure 5. Spatial distribution of KCs with fluorescence transients elicited by cineole (CIN) and MCH. $A, B$, Two flies with three KCs displaying cineole-evoked fluorescence transients. C, D, Two flies with four KCs displaying cineole-evoked fluorescence transients. $E$, Normalized spatial distribution shows three cineole-activated KCs from different flies $(n=6)$ in three tight clusters. $F, G$, Two flies with one KC displaying MCH-evoked fluorescence transients. $H$, I, Two flies with two KCs displaying MCH-evoked fluorescence transients. J, Normalized spatial distribution shows KCs with MCH-evoked fluorescence transients from different flies $(n=5)$ in two tight clusters. Dimensions of the MB are as in $E$. Orientation, frontal views in $A-E$, top views in $F-J$ for better viewing of KCs.

choline receptors (nAChRs) and voltage-

gated $\mathrm{Ca}^{2+}$ channels. All projection neurons are cholinergic in Drosophila (Gorczyca and Hall, 1987; Yasuyama et al., 2002) and most intrinsic KCs express nAChRs (Goldberg et al., 1999; Oleskevich, 1999; Su and O'Dowd, 2003) and voltage-gated $\mathrm{Ca}^{2+}$ channels (Bicker and Kreissl, 1994; Wicher and Penzlin, 1997). Another possible source is $\mathrm{Ca}^{2+}$-induced $\mathrm{Ca}^{2+}$ release from intracellular stores.

In this study, we focused on KCs that showed large fluorescence transients that are significantly above the noise level. Under our experimental conditions, this corresponds to a small subset of KCs. Sampling from a population of $\sim 2800 \mathrm{KCs}$ (four flies, random depths), we counted 58 and $39 \mathrm{KCs}$ with fluorescence transients evoked by EA and BA, respectively. We estimate the overall number of KCs with large fluorescence transients in each brain hemisphere evoked by EA and BA to be 52 and 35, respectively, based on 2500 neurons in each half of the bilateral MB structures. In more extreme cases, cineole and $\mathrm{MCH}$ evoked fluorescence transients in only two to four KCs.

It is possible that there are KCs with small responses to odors that may have been missed because of limited signal-to-noise ratios. Furthermore, G-CaMP is a nonlinear sensor of $\mathrm{Ca}^{2+}$ concentration (Nakai et al., 2001) and neuronal activity and may not detect $\mathrm{Ca}^{2+}$ changes resulting from a single action potential. Using a synthetic $\mathrm{Ca}^{2+}$-sensitive dye, Calcium Green $\left(\mathrm{K}_{\mathrm{D}} \mathrm{s}\right.$ for Calcium Green-1 and -2; 190 and $550 \mathrm{~nm}$, respectively), we have reported previously more broadly distributed activity in the $\mathrm{KC}$ soma layer (Wang et al., 2001). Such synthetic $\mathrm{Ca}^{2+}$ dyes are more sensitive than G-CaMP (T. Pologruto and K. Svoboda, unpublished observations) (Maravall et al., 2000). The large G-CaMP fluorescence transients that we reported here may therefore reveal only a subset of strongly responding units.

However, the sparseness of KCs with odor-evoked G-CaMP fluorescence transients correlates well with the sparseness of $\mathrm{KC}$ spiking responses to odor stimulation in the locust MB (PerezOrive et al., 2002), where on average only $0.7 \%$ of $\mathrm{KCs}$ respond to an odor stimulation, and each responding $\mathrm{KC}$ fires 2.3 spikes on average (Perez-Orive et al., 2002). This correlation suggests that the fluorescence transients in sparse populations of KCs that we observed might represent odor-evoked suprathreshold KC spiking. Furthermore, intracellular recordings in the locust KCs have revealed odor-evoked EPSPs. KCs act as coincidence detectors on projection neuron input, and the conditions for appropriate EPSP summation that leads to $\mathrm{KC}$ firing are rarely met. We speculate that weak fluorescence signals that are possibly missed from 

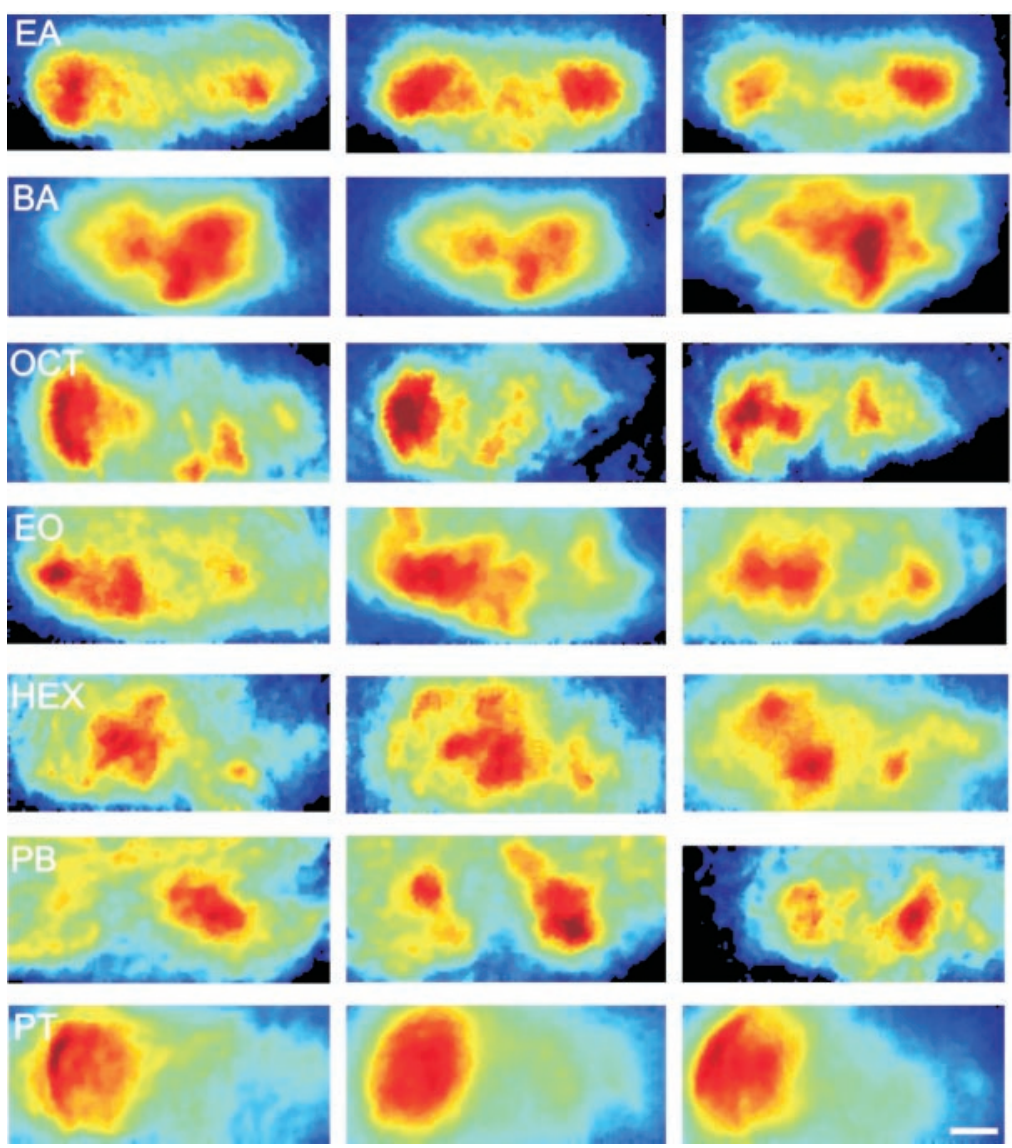

Figure 6. Spatial distribution of odor-evoked G-CaMP activities in the MB calyx (depth, $-50 \mu \mathrm{m}$ ) revealed by a wide-field fluorescence microscope. Each panel was from an individual fly. EA-evoked G-CaMP activities distributed laterally and medially across different individuals, whereas benzaldehyde (BA)-specific activities distributed in the middle region of the calyx. Other odors shown also elicited stereotyped G-CaMP activities in the calyx. All odors were at $10^{-3}$ concentration. E0, Ethyl octanoate; HEX, hexanol; PB, propyl butyrate; PT, propyl tilgate. Orientation is as in Figure 1. Scale bar, $20 \mu \mathrm{m}$.

Table 2. Comparison of odor-evoked G-CaMP activity patterns in calyx regions from individual flies

\begin{tabular}{|c|c|c|c|c|c|c|c|}
\hline & $B A$ & $E A$ & EO & HEX & OCT & $P B$ & PT \\
\hline BA & 0.82 & -0.08 & 0.26 & 0.53 & 0.13 & 0.35 & 0.12 \\
\hline EA & & 0.88 & 0.27 & 0.27 & 0.37 & 0.04 & 0.12 \\
\hline EO & & & 0.72 & 0.55 & 0.25 & 0.21 & 0.42 \\
\hline HEX & & & & 0.81 & 0.12 & 0.24 & 0.33 \\
\hline OCT & & & & & 0.71 & 0.13 & 0.59 \\
\hline PB & & & & & & 0.50 & -0.01 \\
\hline PT & & & & & & & 0.87 \\
\hline
\end{tabular}

Activity patterns for each odor were collected from at least five individual flies. Pearson's product moment correlation coefficients from comparisons of any two individual activity patterns were pooled and averaged. BA, Benzaldehyde; EO, ethyl octanoate; HEX, hexanol; PB, propyl butyrate; PT, propyl tilgate.

our analysis might represent these subthreshold activities. However, the correlation between the G-CaMP fluorescence transients and KC electrical activities can only be established by combining imaging with electrophysiological recordings.

G-CaMP fluorescence transients in different KCs showed diverse degrees of dependency on odor concentration. In some $\mathrm{KCs}$, fluorescence transients were evoked by odors at concentrations spanning several orders of magnitude, whereas in others only by specific concentrations or narrow ranges of concentrations. This finding is consistent with a recent study by Stopfer et al. (2003) in locust, in which they have examined intensity coding in projection neurons and KCs. They found that KCs respond to odors with diverse degrees of concentration invariance, with some units responding to broad ranges of concentration of an

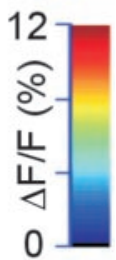

odor, whereas others responded more selectively to specific concentrations. The tuning of KCs to odor concentrations (i.e., selectivity of $\mathrm{KC}$ response to different concentrations of an odor) is interpreted as being a result of specific connections between projection neurons and $\mathrm{KCs}$, oscillatory dynamics, intrinsic properties of KCs, and feed-forward inhibition (PerezOrive et al., 2002; Stopfer et al., 2003).

For odors from different chemical classes such as EA, benzaldehyde, and OCT, there appears to be little overlap between corresponding KC populations with G-CaMP fluorescence transients. This may reflect the fact that these odors activate nonoverlapping sets of ORNs (de Bruyne et al., 2001). Recordings on other odors of dissimilar chemical structures also showed no overlapping KC populations with G-CaMP fluorescence transients. This seems to be different from olfactory cortical neurons in the pyriform cortex of mammals, which have been shown to be less selective to odors (Nemitz and Goldberg, 1983; Schoenbaum and Eichenbaum, 1995). However, different odors were used in these mammalian studies than those used here, complicating direct comparisons between these studies.

The locations of KCs with fluorescence transients evoked by a given odor in MBs are strikingly stereotyped across individuals. In the cases of cineole and $\mathrm{MCH}$, where G-CaMP fluorescence transients were evoked in only a few KCs, individual responsive KCs could be located at similar positions of the MB in different flies with a spatial precision on the order of the size of one or two KC somata. This implies that there may be a genetically determined precise connection between KCs and projection neurons as that found between ORNs and projection neurons (Jefferis et al., 2001), despite the lack of stereotypy of projection neuron arborization patterns in the MB (Marin et al., 2002; Wong et al., 2002). A systematic examination of the spatial distribution of $\mathrm{KC}$ activities in relation to projection neuron activities will reveal the functional connections between projection neurons and KCs.

\section{References}

Bicker G, Kreissl S (1994) Calcium imaging reveals nicotinic acetylcholine receptors on cultured mushroom body neurons. J Neurophysiol 71:808-810.

Bozza TC, Mombaerts P (2001) Olfactory coding: revealing intrinsic representations of odors. Curr Biol 11:R687-R690.

Brand AH, Perrimon N (1993) Targeted gene expression as a means of altering cell fates and generating dominant phenotypes. Development 118:401-415.

Buck LB (2000) The molecular architecture of odor and pheromone sensing in mammals. Cell 100:611-618.

Chess A, Simon I, Cedar H, Axel R (1994) Allelic inactivation regulates olfactory receptor gene expression. Cell 78:823-834. 
Clyne PJ, Warr CG, Freeman MR, Lessing D, Kim J, Carlson JR (1999) A novel family of divergent seven-transmembrane proteins: candidate odorant receptors in Drosophila. Neuron 22:327-338.

Connolly JB, Roberts IJ, Armstrong JD, Kaiser K, Forte M, Tully T, O'Kane CJ (1996) Associative learning disrupted by impaired $\mathrm{G}_{\mathrm{s}}$ signaling in Drosophila mushroom bodies. Science 274:2104-2107.

de Bruyne M, Foster K, Carlson JR (2001) Odor coding in the Drosophila antenna. Neuron 30:537-552.

Denk W, Svoboda K (1997) Photon upmanship: why multiphoton imaging is more than a gimmick. Neuron 18:351-357.

Galizia CG, Sachse S, Rappert A, Menzel R (1999) The glomerular code for odor representation is species specific in the honeybee Apis mellifera. Nat Neurosci 2:473-478.

Gao Q, Yuan B, Chess A (2000) Convergent projections of Drosophila olfactory neurons to specific glomeruli in the antennal lobe. Nat Neurosci $3: 780-785$.

Goldberg F, Grunewald B, Rosenboom H, Menzel R (1999) Nicotinic acetylcholine currents of cultured Kenyon cells from the mushroom bodies of the honey bee Aapis mellifera. J Physiol (Lond) 514:759-768.

Gorczyca MG, Hall JC (1987) Immunohistochemical localization of choline acetyltransferase during development and in Chats mutants of Drosophila melanogaster. J Neurosci 7:1361-1369.

Hildebrand JG, Shepherd GM (1997) Mechanisms of olfactory discrimination: converging evidence for common principles across phyla. Annu Rev Neurosci 20:595-631.

Jefferis GS, Marin EC, Stocker RF, Luo L (2001) Target neuron prespecification in the olfactory map of Drosophila. Nature 414:204-208.

Johnson BA, Woo CC, Leon M (1998) Spatial coding of odorant features in the glomerular layer of the rat olfactory bulb. J Comp Neurol 393:457-471.

Kauer JS, White J (2001) Imaging and coding in the olfactory system. Annu Rev Neurosci 24:963-979.

Korsching S (2002) Olfactory maps and odor images. Curr Opin Neurobiol 12:387-392.

Lendvai B, Stern EA, Chen B, Svoboda K (2000) Experience-dependent plasticity of dendritic spines in the developing rat barrel cortex in vivo. Nature 404:876-881.

Malnic B, Hirono J, Sato T, Buck LB (1999) Combinatorial receptor codes for odors. Cell 96:713-723.

Maravall M, Mainen ZF, Sabatini BL, Svoboda K (2000) Estimating intracellular calcium concentrations and buffering without wavelength ratioing. Biophys J 78:2655-2667.

Marin EC, Jefferis GS, Komiyama T, Zhu H, Luo L (2002) Representation of the glomerular olfactory map in the Drosophila brain. Cell 109:243-255.

Mombaerts P, Wang F, Dulac C, Chao SK, Nemes A, Mendelsohn M, Edmondson J, Axel R (1996) Visualizing an olfactory sensory map. Cell 87:675-686.

Nakai J, Ohkura M, Imoto K (2001) A high signal-to-noise $\mathrm{Ca}(2+)$ probe composed of a single green fluorescent protein. Nat Biotechnol 19:137-141.

Nemitz JW, Goldberg SJ (1983) Neuronal responses of rat pyriform cortex to odor stimulation: an extracellular and intracellular study. J Neurophysiol 49:188-203.

Oleskevich S (1999) Cholinergic synaptic transmission in insect mushroom bodies in vitro. J Neurophysiol 82:1091-1096.

Perez-Orive J, Mazor O, Turner GC, Cassenaer S, Wilson RI, Laurent G (2002) Oscillations and sparsening of odor representations in the mushroom body. Science 297:359-365.

Pologruto TA, Sabatini BL, Svoboda K (2003) ScanImage: flexible software for operating laser scanning microscopes. Biomed Eng Online 2:13.
Rein K, Zockler M, Mader MT, Grubel C, Heisenberg M (2002) The Drosophila standard brain. Curr Biol 12:227-231.

Ressler KJ, Sullivan SL, Buck LB (1994) Information coding in the olfactory system: evidence for a stereotyped and highly organized epitope map in the olfactory bulb. Cell 79:1245-1255.

Rodrigues V (1988) Spatial coding of olfactory information in the antennal lobe of Drosophila melanogaster. Brain Res 453:299-307.

Rubin BD, Katz LC (1999) Optical imaging of odorant representations in the mammalian olfactory bulb. Neuron 23:499-511.

Schoenbaum G, Eichenbaum H (1995) Information coding in the rodent prefrontal cortex. I. Single-neuron activity in orbitofrontal cortex compared with that in pyriform cortex. J Neurophysiol 74:733-750.

Skoulakis E, Acevedo S (2003) Characterization of a novel non-associative behavior, "protection from habituation," defined by D14-3-3zeta mutants.

Spradling AC, Rubin GM (1982) Transposition of cloned P elements into Drosophila germ line chromosomes. Science 218:341-347.

Stocker RF, Singh RN, Schorderet M, Siddiqi O (1983) Projection patterns of different types of antennal sensilla in the antennal glomeruli of Drosophila melanogaster. Cell Tissue Res 232:237-248.

Stopfer M, Jayaraman V, Laurent G (2003) Intensity versus identity coding in an olfactory system. Neuron 39:991-1004.

Su H, O’Dowd DK (2003) Fast synaptic currents in Drosophila mushroom body Kenyon cells are mediated by alpha-bungarotoxin-sensitive nicotinic acetylcholine receptors and picrotoxin-sensitive GABA receptors. J Neurosci 23:9246-9253.

Svoboda K, Denk W, Kleinfeld D, Tank DW (1997) In vivo dendritic calcium dynamics in neocortical pyramidal neurons. Nature 385:161-165.

Technau G, Heisenberg M (1982) Neural reorganization during metamorphosis of the corpora pedunculata in Drosophila melanogaster. Nature 295:405-407.

Vosshall LB, Amrein H, Morozov PS, Rzhetsky A, Axel R (1999) A spatial map of olfactory receptor expression in the Drosophila antenna. Cell 96:725-736.

Vosshall LB, Wong AM, Axel R (2000) An olfactory sensory map in the fly brain. Cell 102:147-159.

Wachowiak M, Cohen LB (2001) Representation of odorants by receptor neuron input to the mouse olfactory bulb. Neuron 32:723-735.

Wang JW, Wong AM, Flores J, Vosshall LB, Axel R (2003) Two-photon calcium imaging reveals an odor-evoked map of activity in the fly brain. Cell 112:271-282.

Wang Y, Wright NJ, Guo H, Xie Z, Svoboda K, Malinow R, Smith DP, Zhong Y (2001) Genetic manipulation of the odor-evoked distributed neural activity in the Drosophila mushroom body. Neuron 29:267-276.

Wicher D, Penzlin H (1997) Ca2 + currents in central insect neurons: electrophysiological and pharmacological properties. J Neurophysiol 77:186-199.

Wong AM, Wang JW, Axel R (2002) Spatial representation of the glomerular map in the Drosophila protocerebrum. Cell 109:229-241.

Yasuyama K, Meinertzhagen IA, Schurmann FW (2002) Synaptic organization of the mushroom body calyx in Drosophila melanogaster. J Comp Neurol 445:211-226.

Yu D, Baird GS, Tsien RY, Davis RL (2003) Detection of calcium transients in Drosophila mushroom body neurons with camgaroo reporters. J Neurosci 23:64-72.

Zou Z, Horowitz LF, Montmayeur JP, Snapper S, Buck LB (2001) Genetic tracing reveals a stereotyped sensory map in the olfactory cortex. Nature 414:173-179. 